\title{
Testicular choriocarcinoma in a case of inguinal hernia: a rare subtype of non-seminomatous mixed germ cell tumour with a misleading presentation requiring a unique treatment approach
}

\author{
S. N. Pravindhira*, P. Prabhakar, R. Kannan, T. Suganthan
}

Institute of General Surgery, Madras Medical College, Chennai, Tamil Nadu, India

Received: 06 December 2020

Revised: 08 December 2020

Accepted: 10 December 2020

\author{
*Correspondence: \\ Dr. SN Pravindhira, \\ E-mail: pravindhira94@gmail.com
}

Copyright: (c) the author(s), publisher and licensee Medip Academy. This is an open-access article distributed under the terms of the Creative Commons Attribution Non-Commercial License, which permits unrestricted non-commercial use, distribution, and reproduction in any medium, provided the original work is properly cited.

\begin{abstract}
Testicular choriocarcinoma is a rare and aggressive variant of non-seminomatous germ cell tumor (NSGCT) with early metastatic spread and dismal prognosis. Early recognition of the diagnosis by elevated $\beta$-human chorionic gonadotropin $(\beta-H C G)$ levels in a young male improves his chances of survival following a radical orchiectomy, which establishes the diagnosis and staging. We report a case of a 21-year old young male presenting with a right groin swelling for 2 months. The clinical history was that of a right inguinal hernia but examination findings suggested a testicular tumor, which was confirmed with an ultrasonograhy and contrast enhanced computed tomography (CECT) of abdomen and scrotum and an elevated $\beta$-HCG of $23,559 \mathrm{IU}$. The metastatic workup was clear and so the patient was taken up for immediate right high inguinal orchidectomy and Lichtenstein tension-free mesh repair. HPE of the postoperative specimen proved a mixed germ cell tumor with choriocarcinoma predominance. Semen analysis and sperm banking were done and the patient was started on chemotherapy and sequential normalization of $\beta$-HCG levels was observed. Choriocarcinoma is an aggressive variant of NSGCT with inherent invasive and metastatic potential rapidly metastasizing to the liver, lung, brain, etc. So a thorough knowledge of the varied clinical manifestations of the choriocarcinoma syndrome ensures early detection even when the presentation is misleading. Treatment is directed towards confirming the diagnosis and staging with histopathology examination and postoperative chemotherapy for persistent normalization of the tumor markers.
\end{abstract}

Keywords: Testicular tumor, Germ cell tumor, Choriocarcinoma, Inguinal hernia, $\beta-\mathrm{HCG}$

\section{INTRODUCTION}

Testicular cancer occurs in 5.7 out of 100,000 men and is the most common solid malignancy occurring in the age group of 15-35 years. ${ }^{1}$ Complete cure is achievable in $95 \%$ of the patients with localized disease and $80 \%$ of those who have metastatic disease. Despite remarkable results with frontline and salvage combination chemotherapy, metastatic testicular cancer remains incurable in approximately $10 \%$ of patients. ${ }^{2}$ Hence early diagnosis, proper clinical staging, and appropriate treatment ensure better prognosis. Two-thirds of the testicular cancers are diagnosed at the localized stage with a 5-year relative survival of $99.0 \%$. Choriocarcinoma is an aggressive subtype of non-seminomatous germ cell tumor (NSGCT) with a far worse prognosis-5 year survival rate of less than $80 \% .^{3}$ It is highly invasive and metastasizes to different cites - lungs, lymph nodes, liver, brain, bones, etc. earlier in its clinical course than most other subtypes of NSGCT. Management of testicular cancer is primarily driven by the clinical stage and whether the tumor is a pure seminoma or a non-seminoma. Cisplatin-based combination 
chemotherapy has made testicular cancer a model for curable neoplasm and is the hallmark for the management of early NSGCT with persistently elevated tumor markers post-orchiectomy, classified as stage IS, due to the high possibility of occult metastasis in this population. ${ }^{1}$ Tumor markers are important for diagnosis, staging, prognostification, assessment of response to therapy, and detection of relapse. The most characteristic presentation of a testicular germ cell tumor is a painless testicular mass. Radical inguinal orchidectomy is both the definitive diagnostic and the initial therapeutic procedure. Surgery should always be performed first unless the clinical situation requires immediate chemotherapy. $4 \mathrm{We}$ report a case of a mixed germ cell tumor with Choriocarcinoma predominance in a 21 -year old male presenting with a right indirect inguinal hernia and a right testicular swelling. This case report highlights the significance of early recognition of elevated $\beta$-HCG levels in a young male presenting with a testicular swelling in a misleading hernia, which directed the treatment approach towards testicular choriocarcinoma.

\section{CASE REPORT}

A 21-year old young male with no other co-morbidities presented with complaints of swelling in the right groin for the past 2 months. The swelling was insidious in onset, gradually progressive in size, aggravated on coughing, associated with a sensation of heaviness in the scrotum, and not associated with pain. No history of fever, burning micturition, vomiting, abdominal pain, abdominal distension, loss of weight, loss of appetite, cough, chest pain, breathlessness and back pain. No history of trauma, previous surgeries, or radiation to the inguinoscrotal regions. No history of cryptorchidism in the past. No history of malignancy in the family members.

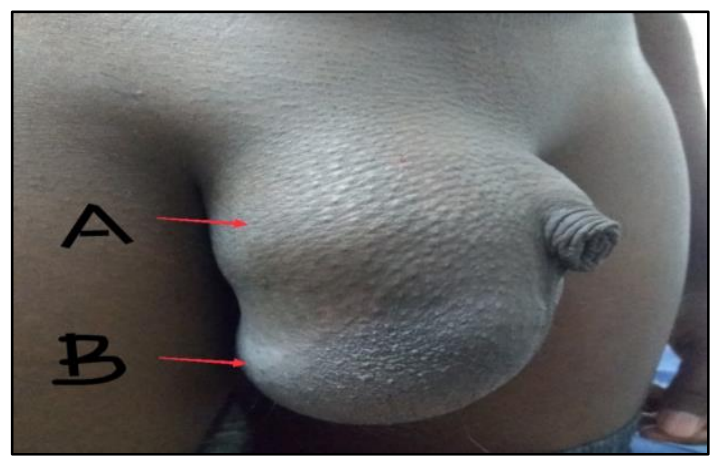

Figure 1: Clinical picture showing two swellings in the right inguino-scrotal. A- right indirect inguinal hernia and $B$ - right testicular carcinoma.

On general examination, his vitals were stable and he was conscious, oriented, afebrile, well built, and wellnourished. Local examination of the right Inguino-scrotal region, with the patient in standing position, showed a pyriform shaped swelling of size $14 \times 7 \mathrm{~cm}$ extending up to the base of the scrotum with a demonstrable expansile cough impulse; skin over the swelling was normal. On palpation with the patient lying down, two separate swellings were made out: A and B. (Figure1) A was a retort-shaped inguinoscrotal swelling of size $9 \times 6 \mathrm{~cm}$, which was non-tender, doughy in consistency with an expansile cough impulse, and was not reducible upon manipulation confirming it to be a right indirect incomplete irreducible inguinal hernia probably omentum as content. B was a spherical shaped pure testicular swelling of size $6 \times 5 \mathrm{~cm}$, which was not tender, not fluctuant, and not transilluminant with a variable consistency and decreased testicular sensation, raising the suspicion of a right testicular tumor. Left inguinoscrotal region was normal.

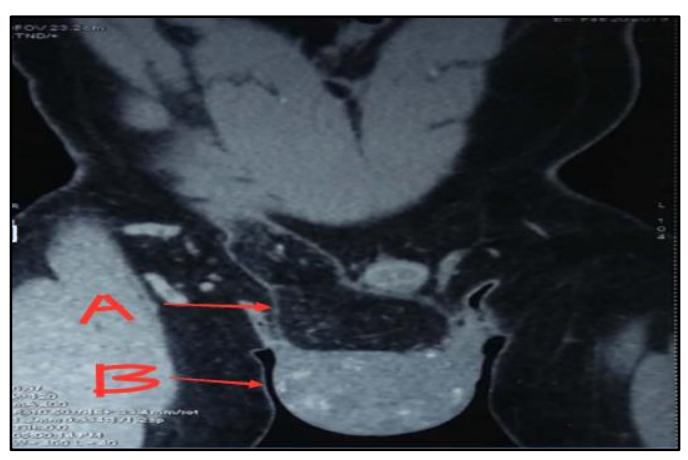

Figure 2: CECT Abdomino-pelvis and scrotum showing: A- right inguinal hernia, B- enlarged right testis with a patchy heterogeneous enhancementsuspicious of a neoplastic lesion.

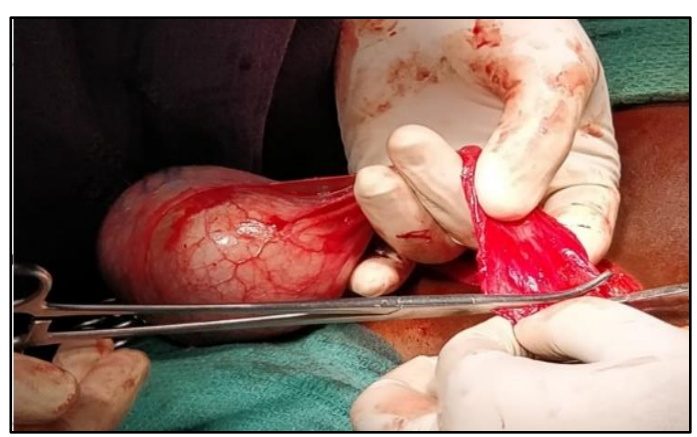

Figure 3 Intraoperative picture of right radical orchiectomy showing an enlarged right testis with increased vascularity. The spermatic chord is seen being clamped high in the inguinal region.

:High-frequency ultrasonography (HF-USG) of the bilateral inguinoscrotal region and USG abdomen revealed right inguinal hernia with omentum as content and a solid mass lesion, with increased vascularity, arising from the lower pole of right testis of size $8 \times 6 \mathrm{~cm}$. CECT abdomen and scrotum revealed an enlarged testis with patchy heterogeneous enhancement suspicious of a neoplastic lesion and no evidence of paraaortic lymphadenopathy or liver metastasis. (Figure 2) The tumor markers panel revealed elevated $\beta$-HCG levels of 23,559 IU (Table 1). CECT chest and CT brain were normal. 
Table 1: The tumor markers panel during the clinical course and management of a choriocarcinoma predominant mixed non-seminomatous germ cell tumor.

\begin{tabular}{|lllllll|}
\hline Tumor markers & Pre-oprative & Post-oprative & Post chemo 1 & Post chemo 2 & Post chemo 3 & Normal \\
\hline $\boldsymbol{\beta}$-HCG & 23559 & 315.50 & 56.4 & 0.59 & 0.21 & $<1.4$ \\
\hline AFP & 80.78 & 6.52 & 6.1 & 5.55 & 3.2 & $<5.5$ \\
\hline LDH & 227 & 201 & 190 & 183 & 181 & $135-214$ \\
\hline
\end{tabular}

The patient was diagnosed with right irreducible indirect inguinal hernia and probable right testicular malignancy and so was taken up for right radical inguinal orchiectomy (Figure 3) and Lichtenstein tension-free hernioplasty. Histopathological examination of the postoperative specimen proved to be a mixed germ cell tumor of the Non-seminoma type: choriocarcinoma predominance $(85 \%)$ with a strong $\beta$-HCG positivity and mature and immature teratoma. (Figure 4 and 5) The patient was discharged without any postoperative complications and was planned for adjuvant chemotherapy on account of the post-operative staging of pT1cNOMOS1; stage - IS.

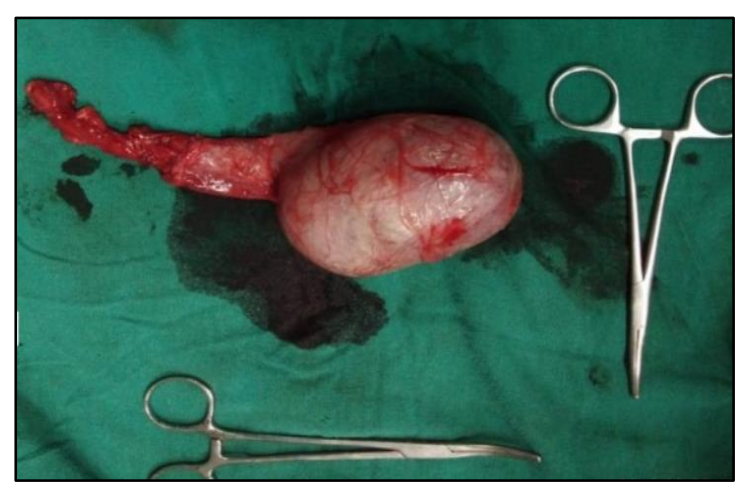

Figure 4: Postoperative radical orchiectomy specimen.

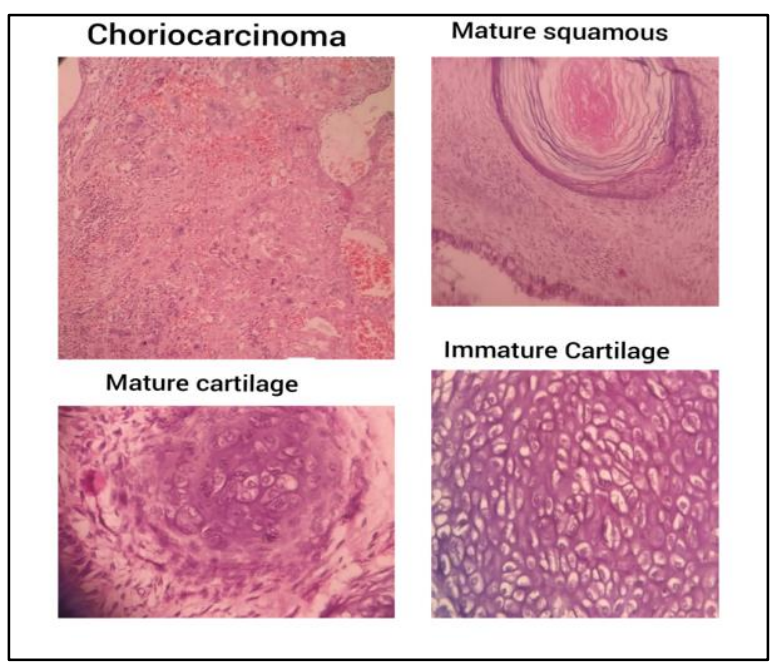

Figure 5: HPE showing features of a mixed nonseminomatous germ cell tumor consisting of Choriocarcinoma \& teratoma- mature and immature.
Since the patient was a young unmarried male, semen analysis and sperm banking was done prior to chemotherapy. The patient was started on BEP regimen: Bleomycin - 30 U IV on days 1,8 and 15 and CDDP 40/40/40/30/70 $\mathrm{mg}$ IV on days 1-5 and Etoposide $200 \mathrm{mg}$ IV on days 1-5 for 3 cycles on an outpatient basis with strict follow-up and sequential monitoring of tumor marker levels which normalized after the 3 cycles (Table 1).

\section{DISCUSSION}

Testicular cancer is the most common solid organ cancer diagnosed in adult men under the age of 34. Overall survival rates are high, with 5-year survival reaching 95\%; however, approximately 400 men die of GCT each year. ${ }^{3,5}$ Over $95 \%$ of testicular tumors originate from germ cells (GCT) and approximately half of the GCT cases are classified as pure seminoma and half are NSGCT which occur in pure or mixed histological forms. The NGCT consists of embryonal carcinoma (11\% pure and $80 \%$ mixed NSGCT cases), choriocarcinoma (0.6\% pure and $33 \%$ mixed NSGCT cases), yolk sac tumors (0.6\% pure and $12 \%$ mixed NSGCT), and teratoma (3.5\% pure and $60 \%$ mixed NSGCT). ${ }^{6}$ Two-thirds $(68 \%)$ of patients present with stage I disease, while $19 \%$ of diagnosed cases are stage II and $12 \%$ are stage $\mathrm{III} .{ }^{3}$ Choriocarcinoma is an aggressive subtype of NSGCT and the patient is prone to present with metastases rather than a testicular mass. The most common presenting symptoms include hemoptysis secondary to pulmonary metastases, back pain secondary to retroperitoneal spread, gastrointestinal bleeding due to gastrointestinal tract metastases, and neurological symptoms that are due to brain metastases. ${ }^{7}$ Yokoi et al recently reported on a review of the literature on choriocarcinoma. ${ }^{8}$ They examined the characteristics of this tumor based on 106 cases of male choriocarcinoma that were reported during the previous 12 years (1995 to 2005). The testis was the most common primary site at $33 \%$ (35/106), followed by the mediastinum, pineal body, gastrointestinal tract, lung and retroperitoneum. Metastasis was seen in $83 \%$ of the patients, with multiple metastases seen in most of the patients. The most common metastatic sites were the lung, liver and brain. The mean age of the males with choriocarcinoma was 36.0 years, and this tumor tended to occur in children and adolescents, where the age of two-thirds of all the cases (69/106) was less than 30 -years old. The prognosis of male choriocarcinoma was 
very poor, with the cumulative survival rate being $30 \%$, and the course of the disease was rapid with the mean survival time being 7.7 months; $23.8 \%$ and $45.4 \%$ of the patients showed 1-month and 6- month mortality, respectively. ${ }^{8}$ Ultrasound (US) of both testes remains the cornerstone of primary imaging in patients with testicular tumors followed by CT of the abdomen and chest as subsequent staging tools for metastatic workup. The correct interpretation of tumor markers before and after orchiectomy in conjunction with CT findings allows correct patient classification according to TNM and Union for International Cancer Control staging. Patients with metastatic disease should be classified according to the International germ cell cancer collaborative group (IGCCCG) to tailor further treatment. ${ }^{9,10}$ Our case had a unique presentation of only a right inguinoscrotal swelling which was misleading to be a right inguinal hernia based on clinical history. Only after a thorough clinical examination was a testicular swelling unmasked and was confirmed by USG. It was suspected to be an NSGCT (testicular choriocarcinoma) based on the considerable elevation of $\beta$-HCG levels in the tumor marker panels and was taken up for urgent right radical inguinal orchiectomy as the imaging modalities provided a clinical staging of localized Germ cell tumor without any lymph node or visceral metastasis. The postoperative HPE and tumor marker panel confirmed a Choriocarcinoma predominant Mixed Germ cell tumor with a TNM staging of stage IS which was managed as follows. Stage IS NSGCT manifest with persistent elevation of tumor markers after radical orchiectomy, without evidence of radiographic disease. Mild elevation of tumor markers after orchiectomy can be a product of nonmalignant causes such as hypogonadism, marijuana use, and hepatobiliary disease, which should be ruled out before proposing any form of adjuvant therapy. Historically, RPLND was recommended in patients who had elevated tumor markers but no obvious evidence of metastatic disease on staging imaging, but subsequent reports observed high relapse rates after primary RPLND. ${ }^{11}$ Saxman et al., suggested that patients with persistently elevated tumors markers should be treated initially with chemotherapy. ${ }^{12}$ Another small retrospective study compared cisplatin-based (16 patients) versus carboplatin-based (4 patients) upfront chemotherapy. Tumor markers returned to normal in all 20 patients, though 3 patients experienced retroperitoneal relapses and 1 died due to the progression of the disease. ${ }^{13}$ In 2008 , Dash et al examined 24 patients with stage IS NSGCT, of which 17 received upfront chemotherapy and three then were treated with elective RPLND. Of the patients who received chemotherapy, 3 (of 14) had a retroperitoneal relapse. All 7 patients who were treated with RPLND relapsed. These data indicate the lack of effectiveness of RPLND alone in stage IS NSGCT. ${ }^{14}$ The NCCN recommends that stage IS NSGCT patients with elevated AFP and/or beta-hCG in the S1 range postoperatively should be treated with chemotherapy for good risk disease. Either 3 cycles of BEP or 4 cycles of EP are preferable treatments because the majority of these patients have disseminated disease. ${ }^{15-17}$ Thus if the tumor markers remain high after orchiectomy; adjuvant chemotherapy remains the hallmark of treatment. After chemotherapy, tumor markers should be assessed until they are normal, as long as they follow their half-life kinetics and no metastases are revealed. Routine contralateral biopsy for diagnosis of carcinoma in situ may be discussed with the patient and is recommended in "high risk" patients (testicular volume $<12 \mathrm{~mL}$, a history of cryptorchidism, defective spermatogenesis, age $<40$ years). The main concern regarding adjuvant radiotherapy is the higher risk of radiation-induced second non-germ cell malignancies. Therefore, adjuvant radiotherapy should no longer be used in young patients. Semen analysis and sperm banking should be offered for all young males before chemotherapy. ${ }^{11-20}$ A testicular prosthesis could be implanted in the scrotum, to provide a normal look. The prosthesis approved for use in the United States is filled with saline (saltwater) and comes in different sizes to match the remaining testicle. ${ }^{13,14}$ Post adjuvant therapy follow-up must be vigilant with assessment of tumor markers, X-ray chest and abdominopelvic CECT. One limitation with current tumor markers is that up to $35 \%$ of relapsing patients have normal levels of tumor markers. The mIR-371a-3p test, a novel biomarker shows remarkable promise in detecting and monitoring the recurrence for NSGCT. ${ }^{11}$

\section{CONCLUSION}

Testicular choriocarcinoma is an aggressive variant of NSGCT that usually presents as a mixed germ cell tumor with early distant metastasis. No delay should be made in proceeding with a radical orchiectomy in a young male with a testicular swelling and elevated $\beta$-HCG levels as early TNM-S staging is vital in identifying the subset of patients with occult metastasis not picked up by imaging modalities. Adjuvant chemotherapy with 3 cycles of BEP regimen is mandatory in stage IS NSGCT and the patient must be in a strict follow up to pick up and cure early relapses to improve outcomes.

\section{Funding: No funding sources Conflict of interest: None declared Ethical approval: Not required}

\section{REFERENCES}

1. Beck, Stephen D W. Management options for stage 1 nonseminomatous germ cell tumors of the testis. Indian journal of urology: IJU : journal of the Urological Society of India. 2010;26(1):72-5.

2. Adra N, Einhorn LH. Testicular cancer update. Clin Adv Hematol Oncol. 2017;15(5):386-96.

3. Bethesda MD. SEER Cancer Stat Facts: Testicular Cancer. National Cancer Institute. https://seer.cancer.gov/statfacts/html/testis.html. Accessed on 02 November, 2020.

4. Oldenburg J, Fosså SD, Nuver J, Heidenreich A, Schmoll HJ, Bokemeyer C, et al. Testicular seminoma and non-seminoma: ESMO clinical 
practice guidelines for diagnosis, treatment and follow-up. Ann Oncol. 2013;24(6):125-32.

5. Siegel RL, Miller KD, Jemal A. Cancer statistics. CA Cancer J Clin. 2018;68:7-30.

6. Stang A, Rusner C, Trabert B. Incidence of testicular tumor subtypes according to the updated WHO classification, North Rhine-Westphalia, Germany, 2008- 2013. Andrology. 2018.

7. Woodward PJ, Heidenreich A, Looijenga LHJ, Oosterhuis JW, McLeod DG, Moller H, et al. Choriocarcinoma. In: Eble JN, Sauter G, Epstein JE, Sesterhenn IA, editors. WHO classification of tumors. Pathology and genetics of tumours of the urinary system and male genital organs. 3rd ed. Lyon: IARC Press. 2004;240-3.

8. Yokoi K, Tanaka N, Furukawa K, Ishikawa N, Seya $\mathrm{T}$, Horiba $\mathrm{K}$, et al. Male choriocarcinoma with metastasis to the jejunum: a case report and review of the literature. J Nippon Med Sch. 2008;75:116-21.

9. Sobin LH, Gospodarowicz MK, Wittekind C, editors. TNM classification of malignant tumors. ed. 7. Oxford, UK: Wiley-Blackwell. 2009;249-54.

10. Mead GM, Stenning SP. The International Germ Cell Consensus Classification: a new prognostic factorbased staging classification for metastatic germ cell tumours. Clin Oncol. 1997;9:207-9

11. Cheriyan SK, Nicholson M, Aydin AM. Current management and management controversies in earlyand intermediate-stage of nonseminoma germ cell tumors. Transl Androl Urol. 2020;9(1):S45-S55.

12. Saxman SB, Nichols CR, Foster RS. The management of patients with clinical stage I nonseminomatous testicular tumors and persistently elevated serologic markers. J Urol. 1996;155:587-9.

13. Culine S, Theodore C, Terrier-Lacombe MJ. Primary chemotherapy in patients with nonseminomatous germ cell tumors of the testis and biological disease only after orchiectomy. J Urol. 1996;155:1296-8.

14. Dash A, Carver BS, Stasi J. The indication for postchemotherapy lymph node dissection in clinical stage IS nonseminomatous germ cell tumor. Cancer. 2008;112:800-5.

15. Lv ZJ, Wu S, Dong P. Clinical outcomes in patients with stage I non-seminomatous germ cell cancer. Asian J Androl. 2013;15:558-63.

16. Mezvrishvili Z, Managadze L. Three cycles of etoposide and cisplatin chemotherapy in clinical stage IS nonseminomatous testicular cancer. Int Urol Nephrol. 2006;38:621-4.

17. National Comprehensive Cancer Network, Clinical Practice Guidelines in Oncology (NCCN Guidelines®), Testicular Cancer, Version 1. 2019. www.nccn.org/professionals/physician_gls/pdf/testi cular.pdf. Accessed on 02 November, 2020.

18. Smith ZL, Werntz RP, Eggener SE. Testicular Cancer: Epidemiology, Diagnosis, and Management. Med Clin N Am. 2018;102:251-264.

19. van den Belt-Dusebout AW, de Wit R, Gietema JA. Treatmentspecific risks of second malignancies and cardiovascular disease in 5-year survivors of testicular cancer. J Clin Oncol. 2007;25:4370-8.

20. Horwich A, Fossa SD, Huddart R. Second cancer risk and mortality in men treated with radiotherapy for stage I seminoma. Br J Cancer. 2014;110:256-63.

Cite this article as: Pravindhira SN, Prabhakar P, Kannan R, Suganthan T. Testicular choriocarcinoma in a case of inguinal hernia: a rare subtype of nonseminomatous mixed germ cell tumour with a misleading presentation requiring a unique treatment approach Int Surg J 2021;8:415-9. 